\title{
Correction to: A formulation of Rényi entropy on $C^{*}$-algebras
}

\section{Farrukh Mukhamedov ${ }^{1} \cdot$ Kyouhei Ohmura $^{2} \cdot$ Noboru Watanabe $^{2}$}

Published online: 15 February 2020

(C) Springer Science+Business Media, LLC, part of Springer Nature 2020

\section{Correction to: Quantum Information Processing (2019), 18:318 https://doi.org/10.1007/s11128-019-2430-3}

The original version of this article unfortunately contained an error in the proof of Theorem 13. The correct transformation of the proof of Theorem 13 is as follows,

$$
\begin{aligned}
(1-\alpha)^{-1} \log \sum_{n, k}\left(\lambda_{n} \mu_{k}^{(n)}\right)^{\alpha} & =(1-\alpha)^{-1} \log \sum_{n}\left(\lambda_{n}\right)^{\alpha} \sum_{k}\left(\mu_{k}^{(n)}\right)^{\alpha} \\
& \geq(1-\alpha)^{-1} \log \sum_{n}\left(\lambda_{n}\right)^{\alpha} \\
& =\mathrm{S}_{\alpha}^{K(\theta)}(\varphi)
\end{aligned}
$$

Publisher's Note Springer Nature remains neutral with regard to jurisdictional claims in published maps and institutional affiliations.

The original article can be found online at https://doi.org/10.1007/s11128-019-2430-3.

$\bowtie \quad$ Kyouhei Ohmura

6317701@ed.tus.ac.jp; ohmura.kyouhei@gmail.com

Farrukh Mukhamedov

far75m@yandex.ru; farrukh.m@uaeu.ac.ae

Noboru Watanabe

watanabe@is.noda.tus.ac.jp

1 Department of Mathematical Sciences, College of Science, United Arab Emirates University, 15551 Al-Ain, United Arab Emirates

2 Department of Information Sciences, Tokyo University of Science, Noda City, Chiba 278-8510, Japan 